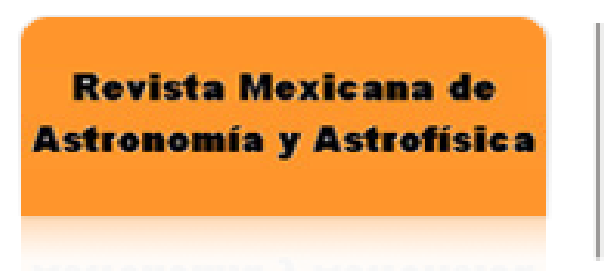

Revista Mexicana de Astronomía y Astrofísica ISSN: 0185-1101

rmaa@astroscu.unam.mx

Instituto de Astronomía

México

Guzmán-Ramírez, L.; Loinard, L.; Gómez, Y.; Morisset, C.

EXPANSION PARALLAX OF THE PLANETARY NEBULA IC 418

Revista Mexicana de Astronomía y Astrofísica, vol. 40, 2011, pp. 141-142

Instituto de Astronomía

Distrito Federal, México

Available in: http://www.redalyc.org/articulo.oa?id=57121297123

How to cite

Complete issue

- More information about this article

Journal's homepage in redalyc.org

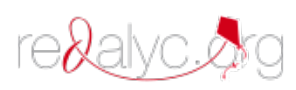

Scientific Information System Network of Scientific Journals from Latin America, the Caribbean, Spain and Portugal Non-profit academic project, developed under the open access initiative 
RevMexAA (Serie de Conferencias), 40, 241-242 (2011)

\title{
EXPANSION PARALLAX OF THE PLANETARY NEBULA IC 418
}

\author{
L. Guzmán-Ramírez, ${ }^{1,3}$ L. Loinard, ${ }^{1}$ Y. Gómez, ${ }^{1}$ and C. Morisset $^{2}$ \\ RESUMEN
}

Presentamos observaciones en dos épocas en radio contínuo de la nebulosa planetaria IC 418, las observaciones están separadas por más de 20 años y fueron realizadas usando el arreglo muy largo, o VLA. Estos datos nos permiten mostrar que la tasa de expansión angular del frente de ionización en IC 418 es $5.8 \pm 1.5$ msa año $^{-1}$. Si la velocidad de expansión del frente de ionización es igual que la velocidad de expansión del gas en la línea de visión, medida usando espectroscopía óptica, entonces para la velocidad de expansión de $30 \mathrm{~km} \mathrm{~s}$, obtenemos una distancia a IC 418 de $1.1 \pm 0.3 \mathrm{kpc}$. Si tomamos en cuenta que el frente de ionización podría estar expandiéndose $20 \%$ más rápido que el gas, entonces la distancia a IC 418 incrementaría a $1.3 \pm 0.4$ kpc.

\section{ABSTRACT}

In this paper, we present radio continuum observations of the planetary nebula IC 418 obtained at two epochs separated by more than 20 years using the Very Large Array. These data allow us to show that the angular

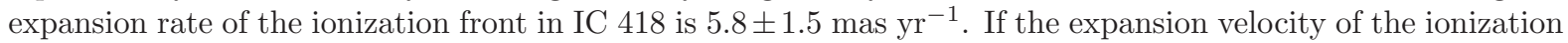
front is equal to the expansion velocity of the gas along the line of sight as measured by optical spectroscopy, then using the value of $30 \mathrm{~km} \mathrm{~s}^{-1}$ for the expansion velocity, we obtained a distance to IC 418 of $1.1 \pm 0.3 \mathrm{kpc}$. If we take into account that the ionization front may be expanding about $20 \%$ faster than the material, then the distance to IC 418 would increase to $1.3 \pm 0.4 \mathrm{kpc}$.

Key Words: planetary nebulae: individual (IC 418) — radio continuum: stars

\section{IC 418}

The Spirograph Nebula (IC 418, G215.2-24.2) has a rather simple morphology: both at optical and radio wavelenghts, it has an elliptical ring shape, with a major axis of $14^{\prime \prime}$ and a minor axis of $10^{\prime \prime}$. It is surrounded by a low-level ionized halo, which is itself enshrouded in a neutral envelope with an angular size of about $2^{\prime \prime}$ (Taylor \& Pottasch 1987; Taylor et al. 1989). Widely discrepant estimates of the distance to the planetary nebula (PN) IC 418 have been obtained using different statistical methods. To our knowledge, the shortest distance ever proposed is $360 \mathrm{pc}$ (Acker 1978), whereas the largest one is $5.74 \mathrm{kpc}$ (Phillips \& Pottasch 1984). In recent years, the most popular value appears to have been 1 kpc (Meixner et al. 1996; Pottasch et al. 2004), although the reason for this is not entirely clear.

\footnotetext{
${ }^{1}$ Centro de Radioastronomía y Astrofísica, Universidad Nacional Autónoma de México, Apdo. Postal 3-72, 58090, Morelia, Michoacán, México.

2Instituto de Astronomía, Universidad Nacional Autónoma de México, 04510 México D.F., México.

${ }^{3}$ Jodrell Bank Observatory, University of Manchester, School of Physics and Astronomy, Alan Turin Building M13 9PL, UK (lizette.ramirez@postgrad.manchester.ac.uk).
}

\section{RESULTS AND DISCUSSION}

The data were taken using the Very Large Array (VLA) of the $\mathrm{NRAO}^{4}$ at $6 \mathrm{~cm}(5 \mathrm{GHz})$ in its second most extended (B) configuration on June 28, 1986 (1986.49) and November 6, 2007 (2007.85). This is a time separation of $21.36 \mathrm{yr}$. The resulting images are shown in Figure 1.

The difference image between the two epochs, shown in the bottom left panel of Figure 1 was produced following Guzmán et al. (2006). We estimated the expansion rate of the nebula by comparing the difference image of the data with the model. To generate the model difference, we image the data twice. The first image was made using a fixed pixel size of 0 !! 1 , whereas the second one was made using pixel sizes of $(1+\epsilon) \times 00^{\prime \prime} 1$, with $\epsilon \geq 0$, but $\epsilon \ll 1$. See Guzmán et al. (2009) for more detail. To identify the best model, we repeated the procedure described above for a set of values of $\epsilon$. For each value, we compared the model difference with the real difference. The best model clearly corresponds to the situation where the two differences are as similar to each other as possible. The best value for $\epsilon$ that we obtained was $\epsilon=0.018 \pm 0.005$. Using the image of the second

${ }^{4}$ The National Radio Astronomy Observatory is operated by Associated Universities, Inc., under a cooperative agreement with the National Science Foundation. 

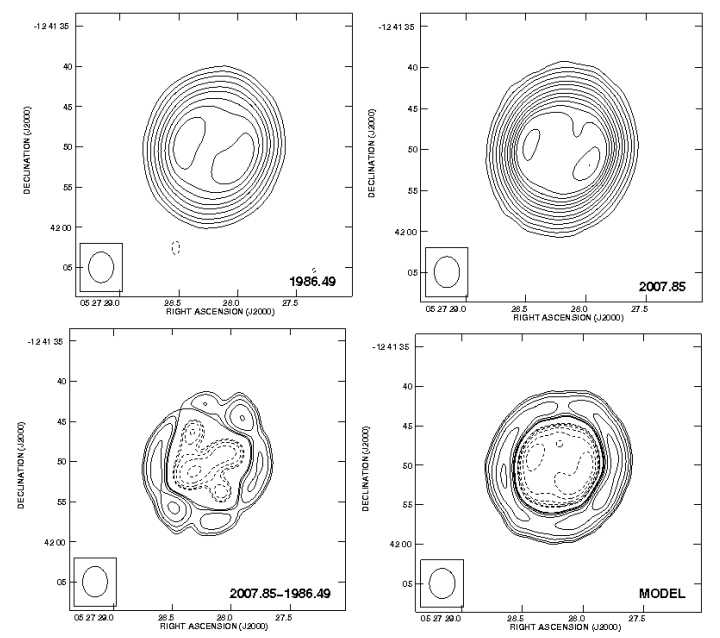

Fig. 1. Top: contour images of the $6 \mathrm{~cm}$ emission form IC 418 for 1986.49 (left) and 2007.85 (right). The contours are $-5,5,10,30,50,60,80,100,150,200,250$ and 300 times $350 \mu \mathrm{Jy}$, the average rms noise of the images. Bottom: contour images of the $6 \mathrm{~cm}$ difference image (left) and of the model (right) obtained as described in the text. The contours are $-20,-15,-10,-7,-5,-4$, $4,5,7,10,15$ and 20 times $460 \mu \mathrm{Jy}$, the rms noise of the difference image.

epoch we estimated the radius of maximum emission, $\theta$ to be $6 . .700 \pm 0$ ". 006 . Using this value and $\epsilon$ 's value we can calculate the angular expansion rate, $\dot{\theta}$ to be

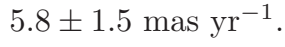

To deduce the distance from the angular expansion rate calculated above, one must know the physical velocity $v_{\exp }$ at which the ionization front is expanding: $[\mathrm{D} / \mathrm{pc}]=211\left[v_{\exp } / \mathrm{km} \mathrm{s}^{-1}\right]\left[\dot{\theta} / \mathrm{mas} \mathrm{yr}^{-1}\right]^{-1}$.

Traditionally, $v_{\exp }$ has been estimated using high spectral resolution observations of some emission lines and assuming a relation between the shape of the line profile and the movement of the emitting gas. For this nebula IC 418, we used the high resolution spectra of $\mathrm{H} \beta$, [NII] and [OIII] lines published by Gesicki et al. (1996). Using a detailed three-dimensional photoionization model of the nebula, Morisset \& Georgiev (2009) reproduced these three profiles using a $V \propto R^{4}$ expansion law. According to this model, the expansion velocity near the outer edge of the nebula (where we detect the expansion at radio wavelengths) is $30 \mathrm{~km} \mathrm{~s}^{-1}$. Using this value we can calculate the distance to IC 418, we obtained a distance of $1.1 \pm 0.3 \mathrm{kpc}$.

To calculate this distance we have assumed that the velocity of the ionization front (pattern velocity) is the same as the Doppler velocity of the material
TABLE 1

PHYSICAL PARAMETERS OF IC 418

\begin{tabular}{cc}
\hline Parameter & Value \\
\hline$t_{\mathrm{dyn}}$ & $1200 \mathrm{yr}$ \\
$\mathrm{EM}$ & $(5.05 \pm 0.01) \times 10^{-6} \mathrm{~cm}^{-6} \mathrm{pc}$ \\
$n_{e}$ & $(6.2 \pm 1.7)(d / 1.1 \mathrm{kpc})^{-0.5} \times 10^{3} \mathrm{~cm}^{-3}$ \\
$M_{i}$ & $(8.7 \pm 2.4)(d / 1.1 \mathrm{kpc})^{2.5} \times 10^{-2} M_{\odot}$ \\
\hline
\end{tabular}

itself, deduced form spectral lines. For conditions appropriate for planetary nebulae (PNe), Mellema (2004) showed that the ionization front tends to expand somewhat faster than the material. Using their Figure 5, we estimated that the expansion velocity of the ionization front in IC 418 is $1.2 \pm 0.1$ faster than the expansion velocity of the gas. Taking this effect into account leads to a distance to IC 418 of $1.3 \pm 0.4 \mathrm{kpc}$.

Using the distance value, we can derive the physical parameters of the nebula like the electron density $\left(n_{e}\right)$ and the mass of ionized gas $\left(M_{i}\right)$ and using the angular expansion rate we can derive the dynamical age of the nebula $\left(t_{\mathrm{dyn}}\right)$. All these parameters together with the emission measure (EM) calculation are listed in Table 1.

\section{CONCLUSIONS}

In this document, we presented observations of the $6 \mathrm{~cm}$ radio continuum emission from the well studied PN IC 418 obtained at two epochs separated by more than 20 years. This data allowed us to detect the angular expansion of the nebula, and to estimate its distance. Depending on the assumption made on the relative velocity of the matter and of the ionization front, we obtained a distance of $1.1 \pm 0.3 \mathrm{kpc}$, or $1.3 \pm 0.4 \mathrm{kpc}$. Using this distance we derived the physical parameters of the planetary nebula IC 418 .

\section{REFERENCES}

Acker, A. 1978, A\&AS, 33, 367

Gesicki, K., Acker, A., \& Szczerba, R. 1996, A\&A, 309, 907

Guzmán, L., Gómez, Y., \& Rodríguez, L. F. 2006, RevMexAA, 42, 127

Guzmán, L., et al. 2009, AJ, 138, 46

Meixner, M., et al. 1996, A\&A, 313, 234

Mellema, G. 2004, A\&A, 416, 623

Morisset, C., \& Georgiev, L. 2009, A\&A, 507, 1517

Phillips, J. P., \& Pottasch, S. R. 1984, A\&A, 130, 91

Pottasch, S. R., et al. 2004, A\&A, 423, 593

Taylor, A. R., \& Pottasch, S. R. 1987, A\&A, 176, L5

Taylor, A. R., Gussie, G. T., \& Goss, W. M. 1989, ApJ, 340,932 